\title{
Levantamento malacológico, identificação de moluscos e cercárias transmissoras de Schistosoma mansoni do município de Arcos - MG.
}

\author{
Malacological survey, identification of molluscs and surroundings, \\ transmitters of Schistosoma mansoni in the municipality of Arcos - Minas \\ Gerais
}

Gabrielly Costa Campos ${ }^{1}$; Rafaela Faria Rodarte Ribeiroํ; Egídia Carolina de Oliveira ${ }^{1}$; Jordânia Costa Pinto ${ }^{1}$; Mariana Teixeira de Faria ${ }^{2}$; José Carlos Leal ${ }^{2}$; Ivani Pose Martins ${ }^{2}$; Fernando Sérgio Barbosa $^{2}$.

${ }^{1}$ Centro Universitário de Formiga - MG, Unifor, Formiga, Minas Gerais, Brasil.

\section{Resumo}

Introdução: A esquistossomose é considerada um problema de saúde pública no Brasil. Sua transmissão ocorre em ambientes hídricos dulcícolas habitados por moluscos das espécies Biomphalaria glabrata, B. straminea e B. tenagophila, e sua identificação é relevante para o controle e epidemiologia dessa parasitose. Objetivos: O presente estudo buscou identificar as áreas de risco para transmissão de Schistosoma mansoni no município de Arcos-MG, por meio do levantamento malacológico e caraterização das cercárias emergentes. Metodologia: Os moluscos foram coletados manualmente com o auxílio de pinça e peneira, colocados em caixas térmicas e transportados para análise no laboratório de Microscopia do UNIFOR-MG. Em laboratório foi realizada a mensuração e identificação dos moluscos pelas características taxonômicas. Em seguida, as espécies foram separadas e foi realizada a caracterização das cercárias emergidas em microscópio. Resultados: Foram coletados 6445 moluscos e identificadas quatro espécies, Physa marmorata, Biomphalaria glabrata, Lymnaea columella e B. tenagophila. Destes exemplares, 27 estavam infectados por larvas de trematódeos, $11,1 \%$ da espécie $B$. glabrata e $88,9 \%$ P. marmorata. Dos exemplares de $B$. glabrata 7,2\% estavam positivos para cercárias de $S$. mansoni e 3,7\% para cercárias de Echinostoma $s p$. Em relação à Physa marmorata 55,6\% estavam positivos para cercárias do tipo Xifideocercária e 33,3\% para cercárias Echinostoma sp. Conclusão: O estudo da malacofauna foi extremamente importante, pois foram identificados moluscos transmissores da esquistossomose, o que colocava a área em risco para transmissão da esquistossomose, além do encontro de moluscos de outras espécies, liberando larvas de outras espécies de trematódeos, representando assim, riscos para a transmissão de doenças de interesse médico e veterinário.

Palavras-chave: Cercárias; Esquistossomose; Levantamento malacológico. 


\section{Abstract}

Introduction: Schistosomiasis is considered a public health problem in Brazil, its transmission occurs in sweet water environments inhabited by mollusks of the species Biomphalaria glabrata, B. straminea and B. tenagophila, being its relevant identification for the control and epidemiology of this parasitose. Objectives: The present study aimed to identify the risk areas for transmission of Schistosoma mansoni in the municipality of Arcos-MG, through the malacological survey and characterization of emergent cercariae. Methodology: The molluscs were manually collected with the aid of tongs and sieves, placed in polystyrene boxes and transported for analysis in the Microscopy laboratory of UNIFOR-MG. In the laboratory the measurement and identification of the molluscs through the taxonomic characteristics was carried out. Then the species were separated and the characterization of the cercariae emerged under a microscope. Results: 6445 mollusks and identified four species were collected, Physa marmorata, Biomphalaria glabrata, Lymnaea columella and B. tenagophila. You gave copies 27 were positive for larvas of trematodes, being 11,1\% of the species B. glabrata, where 7,2\% were positive for cercárias of S. mansoni and 3,7\% for cercárias of Echinostoma sp. Other 88,9\% mollusks of the species Physa marmorata were infected, being 55,6\% for cercárias of the type Xifideocercária and 33,3\% for cercárias Echinostoma sp. Conclusion: The study of the malacofauna was extremely important, because mollusks transmitting schistosomiasis were identified, placing the area at risk for the transmission of schistosomiasis, in addition to the encounter of molluscs, other molluscs releasing larvae from other species of trematodes that represent risks for the transmission of diseases of medical and veterinary interest.

Keywords: Cercariae; Schistosomiasis; Malacological Survey.

\section{Introdução}

A esquistossomose é uma doença tropical negligenciada, ocasionada por parasitos do gênero Schistosoma, várias espécies como $S$. japonicum, $S$. haematobium, S. intercalatum, S. mekongi, S. mansoni já foram descritas, porém a de interesse médico no Brasil é a $S$. mansoni, por ser a única responsável pelos casos de infecção no país ${ }^{1}$.

A ocorrência da esquistossomose está ligada principalmente a regiões de baixa renda, falta de saneamento básico e escolaridade, fato que ocorre na maioria dos países em desenvolvimento ${ }^{2,3}$. Segundo a Organização Mundial da Saúde ${ }^{4}$, cerca de 240 milhões de pessoas estão infectadas e 700 milhões vivem sob área de risco.

Essa parasitose é um grande problema de saúde pública no Brasil, e é encontrada em várias regiões brasileiras. Devido ao crescimento populacional e à expansão das áreas urbanas, aumentam os riscos de expansão geográfica da doença. Estima-se que 27 milhões de pessoas vivem sob áreas de risco no país ${ }^{5}$. O estado de Minas Gerais é considerado uma das regiões endêmicas da esquistossomose, por apresentar vários casos da doença, por isso é de grande importância a realização de pesquisas e projetos voltados ao combate da parasitose ${ }^{6}$.

Para que a parasitose se instale em determinado local, é necessária a presença de moluscos, vetores da doença, como também a falta de saneamento básico, a falta de instrução e conhecimento da população sob a doença e, ainda, como prevení-la ${ }^{7}$.

A transmissão ocorre em locais de água doce habitados por moluscos das espécies $B$. glabrata, $B$. straminea e B. tenagophila ${ }^{8}$. Durante o contato com a água, as formas larvais que são liberadas pelos moluscos podem penetrar na pele do homem, atingindo o sistema vascular, local onde os parasitos adultos se desenvolvem ${ }^{9}$.

Considerando que a transmissão e manutenção da esquistossomose é possível somente com a presença dos moluscos suscetíveis, o estudo da malacofauna auxilia na identificação de uma área de risco para transmissão da doença. Além disso, a identificação de outras espécies de moluscos são de grande importância para estudos da biologia, pois várias espécies de trematódeos estão 
envolvidas na transmissão de doenças para animais silvestres e domésticos. Assim, o monitoramento e identificação da malacofauna se torna relevante para orientar no controle e combate a infecção, pois corroboram que áreas de risco sejam evidenciadas e evitadas $^{10,11,12}$.

Visando a isso, o presente estudo realizou a coleta e identificação de moluscos em locais hídricos no município de Arcos-MG, onde a população utiliza a água para pesca e/ou lazer, com o objetivo de realizar a análise da malacofauna, identificando as espécies de moluscos encontradas, observando a ocorrência de cercárias nos moluscos coletados, para averiguação das áreas de risco para transmissão da esquistossomose.

\section{Metodologia}

$\mathrm{O}$ estudo foi realizado na cidade de Arcos-MG ( $\left.20^{\circ} 16^{\prime} 55^{\prime \prime} \mathrm{S} 45^{\circ} 32^{\prime} 22^{\prime \prime} \mathrm{W}\right)$ localizada no Centro-Oeste Mineiro (FIGURA 1). Segundo o $\mathrm{IBGE}^{13}$, o município possui uma área territorial de $514,4 \mathrm{Km}^{2}$. A população estimada é de aproximadamente $39.793^{14}$.

$$
\text { O clima é predominantemente tropical, e }
$$
apresenta duas estações bem estabelecidas: verão chuvoso, entre agosto e fevereiro e inverno seco, de maio a setembro. A temperatura média anual é de $21,3^{\circ} \mathrm{C}$. Tem uma pluviosidade média anual de $1367 \mathrm{~mm}^{14}$

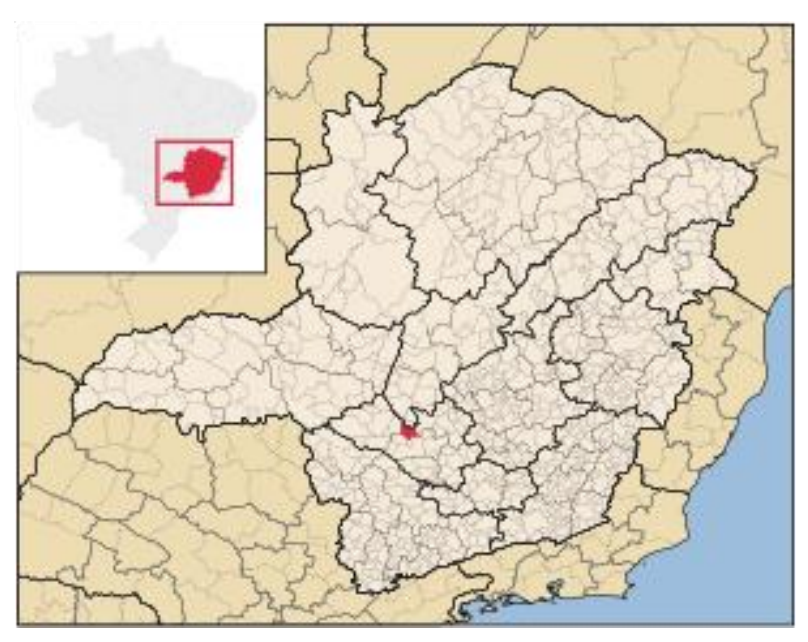

FIGURA 1: Mapa identificando o município de Arcos-MG, onde foram realizadas as coletas malacológicas. Google Earth.

Os locais de coletas foram selecionados de acordo com as características do ambiente como vegetação, acessibilidade e ambientes lênticos (FIGURA 2A, 2B, 2C). As coletas foram realizadas entre o período de junho de 2017 a junho de 2018, com o auxílio de uma peneira confeccionada com nylon e pinças metálicas. Os moluscos coletados foram colocados em sacos plásticos, identificados, acondicionados em caixas térmicas e transportados para o laboratório de Microscopia do Centro Universitário de Formiga (UNIFOR-MG). 

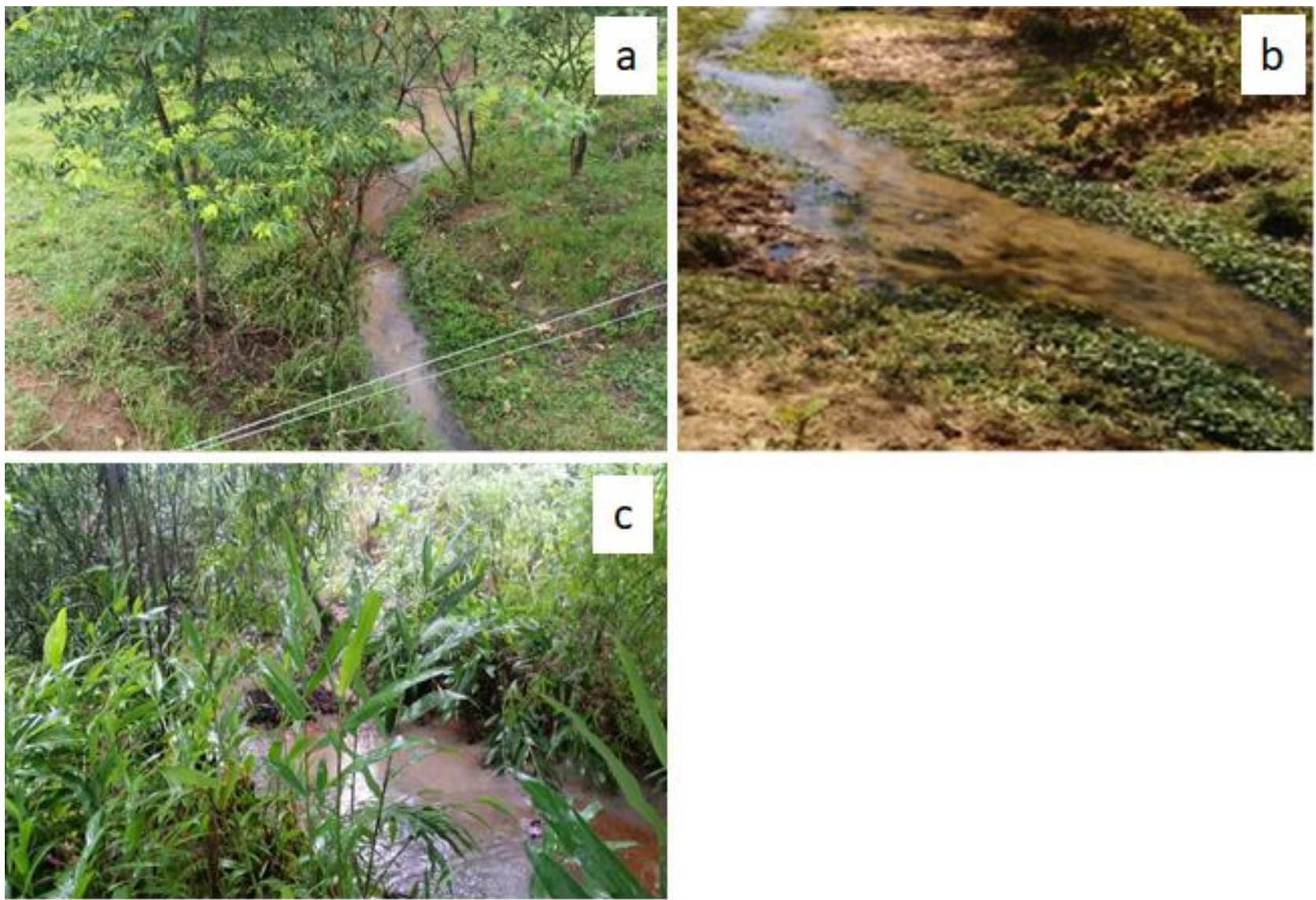

FIGURA 2A: Coleta realizada na lagoa da comunidade Boca da Mata, distrito pertencente ao município de Arcos-MG.

FIGURA 2B: Coleta realizada no riacho situado na fazenda Igarapé, localizada no município de Arcos-MG.

FIGURA 2C: Coleta realizada no lago situado na fazenda Bonita, localizado no município de Arcos-MG. Arquivo pessoal

No laboratório, foi realizada a triagem e identificação, por meio das características taxonômicas dos moluscos, por meio de parâmetros conquiológicos e morfológicos15. Para a verificação da emergência de cercárias os moluscos foram colocados individualmente em placas de cultura de 24 poços, contendo $5 \mathrm{ml}$ de água isenta de cloro e deixadas durante a noite. Na manhã seguinte, foram analisados, antes e após duas horas de fotoestimulação artificial, para observação de cercárias emergentes. As cercárias que emergiram dos moluscos foram concentradas, posteriormente pipetadas, colocadas entre lâmina e lamínulas e analisadas microscopicamente em microscópio com auxílio de corantes vitais (Vermelho Neutro e sulfato azul do Nilo a $0,05 \%)^{16}$.

Para a identificação das espécies de cercarias, as amostras foram comparadas com as descrições e chaves de identificação propostas pelos autores ${ }^{17,18,19}$.
Os moluscos negativos foram macerados em placa de vidro, analisados em microscópio para confirmação da ausência de larvas de trematódeos e posteriormente foram desprezados ${ }^{5}$.

\section{Resultados}

Foram coletados e examinados 6445 exemplares de moluscos e identificadas quatro espécies: 43,21\% exemplares de Physa marmorata, 33,02\% de Biomphalaria glabrata, 13,44\% de Lymnaea columella e 10,33\% exemplares de B. tenagophila (FIGURA 3). 


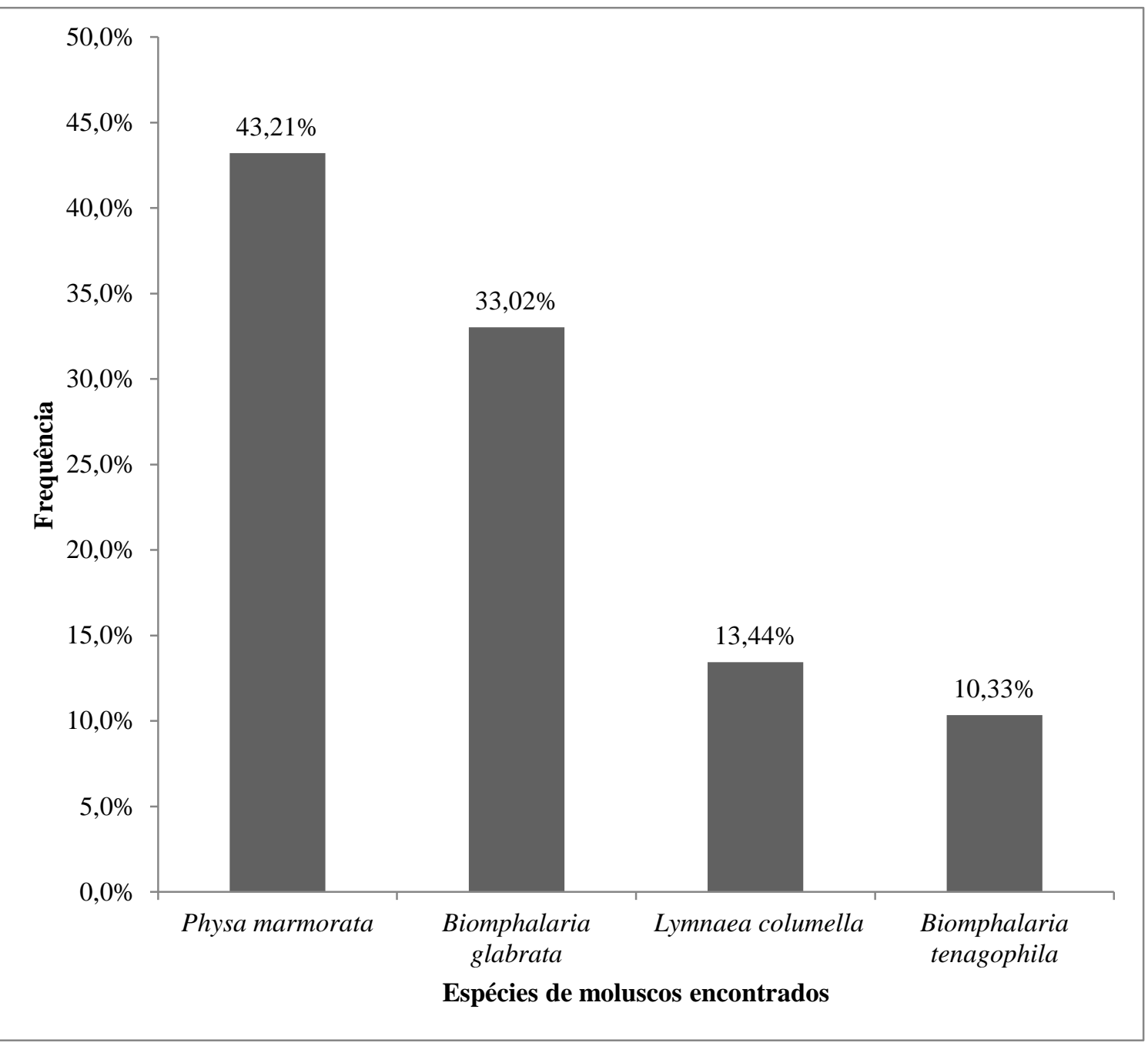

FIGURA 3: Exemplares de moluscos coletados em levantamento realizado em locais hídricos no município de Arcos-MG

Do total de moluscos coletados, 27 estavam infectados por larvas de trematódeos, $11,1 \%$ da espécie $B$. glabrata e $88,9 \%$ P. marmorata. Dos exemplares de B. glabrata $7,2 \%$ estavam positivos para cercárias de S. mansoni e
3,7\% para cercárias de Echinostoma sp. Em relação à Physa marmorata 55,6\% estavam positivos para cercárias do tipo Xifideocercária e 33,3\% para cercárias Echinostoma sp. (FIGURA 4). 


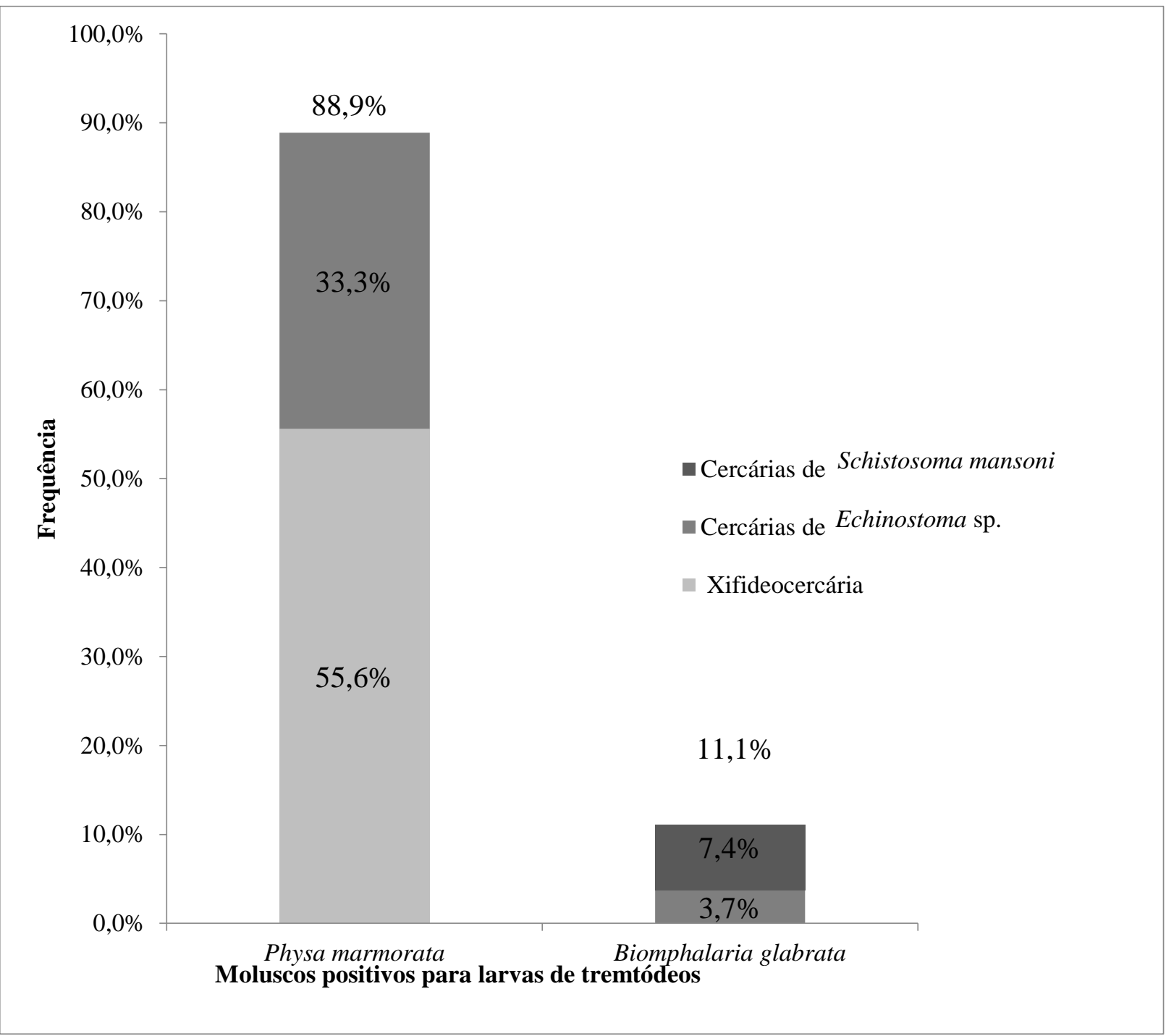

FIGURA 4: Espécies de moluscos encontrados naturalmente infectados por larvas de trematódeos.

As cercárias de $S$. mansoni são caracterizadas por apresentarem cauda longa e bifurcada com comprimento aproximadamente três vezes maior que sua bifurcação e ventosa ventral bem desenvolvida (FIGURA 5A).
As cercárias do tipo Xifideocercária são caracterizadas por possuírem um estilete na ventosa oral (FIGURA 5B). Já as cercárias Echinostoma sp. são caracterizadas por possuir um colar de espinhos que circundam a ventosa oral (FIGURA 5C). 

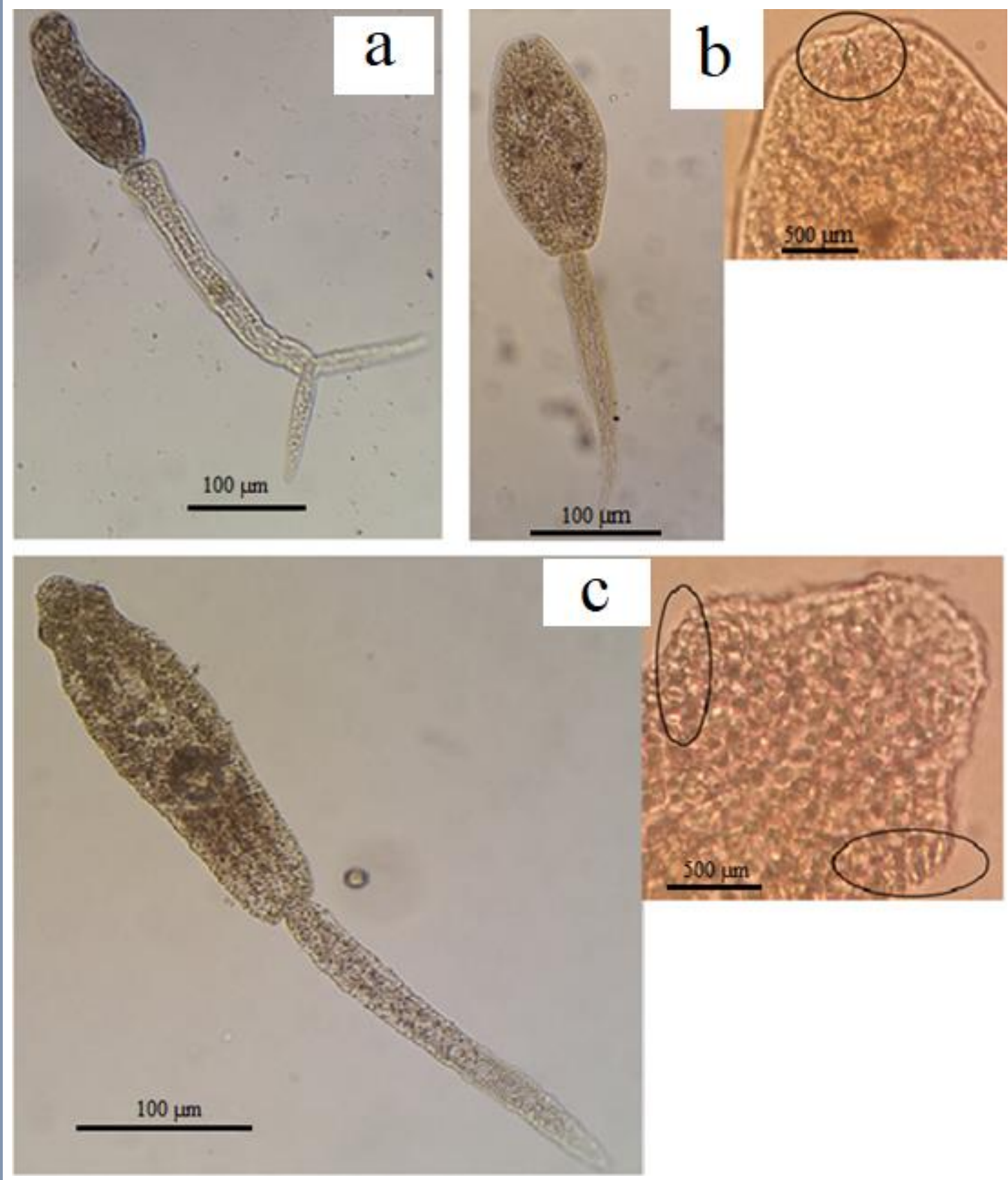

FIGURA 5A: Cercária da espécie do $S$. mansoni que emergiram em moluscos da espécie B. glabrata.

FIGURA 5B: Cercárias do tipo Xifideocercária que emergiram em moluscos da espécie $P$. marmorata, em destaque demonstrando o estilete presente na ventosa oral.

FIGURA 5C: Cercárias da espécie Echinostoma sp. que emergiram em moluscos das espécies B. glabrata e P. marmorata, em destaque o colar de espinhos que circundam a ventosa oral.

A expansão de $S$. mansoni ocorre devido às migrações humanas, entretanto para o estabelecimento da esquistossomose em uma determinada região dependerá principalmente das espécies de moluscos existentes, do grau de saneamento básico, das condições climáticas e do contato da população humana com os criadouros de moluscos ${ }^{20,21}$.

O presente estudo demonstra que há presença de moluscos do gênero Biomphalaria na região analisada, o que coloca esses locais em risco para a transmissão da esquistossomose, além disso, outras espécies de moluscos 
de relevância médico-veterinária foram encontradas, contribuindo para o estudo da malacofauna no município.

A presença de moluscos Biomphalaria também tem sido relatada em outras regiões de Minas Gerais por diversos autores. Com a presença desse molusco, a área passa a ser considerada como de risco, em relação a transmissão da esquistossomose ${ }^{5,6,22}$.

Neste estudo do molusco da espécie B. glabrata foram encontradas naturalmente infectados por $S$. mansoni, esse fato é preocupante, pois de apenas um molusco pode emergir milhares de cercárias e estas são capazes de manter o ciclo da parasitose em uma determinada área, além disso, a presença de moluscos infectados por $S$. mansoni demonstra que há indivíduos parasitados nessa região. Foram descritos ainda que somente a presença de moluscos suscetíveis à infecção, mesmo que negativos para larvas de trematódeos, o local passa a ser considerado como uma área de risco, pois podem se tornar focos de transmissão da esquistossomose $\mathrm{e}^{10}$.

Vários autores relatam uma maior suscetibilidade do trematódeo $S$. mansoni pela espécie de molusco $B$. glabrata e que essa espécie é responsável pela transmissão da esquistossomose em várias cidades mineiras ${ }^{5 ; 8 ; 23}$. A $B$. tenagophila é considerada como a segunda espécie mais suscetível para o desenvolvimento de S. mansoni e, quando infectada experimentalmente em laboratório, tem apresentado alto grau de adaptação por diversas cepas ${ }^{8}$. Já B. straminea, embora a menos suscetível, seja a espécie responsável pela transmissão e manutenção da doença, principalmente na região nordeste do país ${ }^{24}$.

Durante o levantamento realizado para o presente estudo, também foram identificadas outras espécies de moluscos como P. marmorata e L. columella, este último hospedeiro intermediário de Fasciola hepatica. Embora não haja casos notificados de Fasciliose na região, a presença dos moluscos L. columella demonstra que, na região estudada, podem ocorrer focos de transmissão da doença $^{25}$. Já o molusco $P$. marmorata é responsável por albergar formas evolutivas de parasitos que podem transmitir doenças para outras espécies de animais ${ }^{26}$.
Vários são os tipos de cercárias de trematódeos relatados no Brasil, porém o impacto ecológico das infecções em populações de moluscos por esses trematódeos ainda é pouco conhecido e isso está amplamente aberto a investigações de campo e que interessa às eventuais tentativas de controle ou erradicação das populações naturais dos moluscos e dos parasitos por eles transmitidos $27,28,29$.

No presente estudo, outras formas larvais de trematódeos também foram caracterizadas, como as cercárias Echinostoma sp. e Xifideocercárias encontradas em moluscos P. marmorata. Nos moluscos da espécie $B$. glabrata além de cercárias S. mansoni, também foram encontradas cercárias de Echinostoma sp., portanto estudos complementares devem ser realizados com o intuito de determinar qual a sua importância para a parasitologia veterinária ${ }^{30,31,32}$.

\section{Conclusão}

As áreas estudadas apresentam condições ecológicas para a expansão da esquistossomose, pois foram encontrados moluscos do gênero Biomphalaria sp., o que chama atenção para os programas de saúde pública do município, uma vez que esses exemplares apresentaram naturalmente infectados por larvas da esquistossomose e esses locais são coleções hídricas utilizadas pela população local, o que aumenta as chances de contaminação pela população.

Estudos nessa área são relevantes e devem ser incentivados, pois além de ajudar na determinação de locais de riscos para a transmissão de algumas parasitoses, auxiliam na identificação da malacofauna, no estudo do ciclo biológico e na interação entre parasito e hospedeiro.

\section{Declaração de Conflito de Interesses}

Os autores do artigo afirmam que não houve nenhuma situação de conflito de interesse, tais como propostas de financiamento, emissão de pareceres, promoções ou participação em comitês consultivos ou diretivos, entre outras, que pudessem influenciar no desenvolvimento do trabalho. 


\section{Referências}

1 KATZ, N.; ALMEIDA, K. Esquistossomose, xistosa, barriga d'água. Ciência e Cultura, São Paulo, v. 55, n. 1, p. $38-41,2003$.

2 MINISTÉRIO DA SAÚDE. Esquistossomose.

Situação epidemiológica - Dados. Disponível em:<

http://portalsaude.saude.gov.br/index.php/o-

ministerio/principal/leia-mais-o-ministerio/656-

secretaria-svs/vigilancia-de-a-a-

z/esquistossomose/11244-situacao-epidemiologica-

dados>. Acesso em: 18 fev. 2018.

3 BORDA, C. E.; REA, M.J.F. Intermediate and definitive hosts of Schistosoma mansoni in Corrientes province, Argentina. Mem Inst Oswaldo Cruz, Rio de Janeiro, v. 101(Suppl. I): 233-234, 2006.

4 WHO, WORD HEALTH ORGANIZATION.

Schistosomiasis. The disease. Disponível em: <http://www. who.int/schistosomiasis/disease/en/>. Acesso em: 17 fev. 2018.

5 MELO, A. G. S de.; MELO, C. M, de.; OLIVEIRA, C. C. C. da.; OLIVEIRA, D. S.; SANTOS, V. B.; JERALDO, V. L. S. de. Esquistossomose em área de transição rural-urbana: reflexões

epidemiológicas. Ciência, Cuidado e Saúde, v. 10, n. 3, p. 506-513, 2011.

6 MASSARA, C. L.; CARVALHO, O. S.; CALDEIRA, R. L.; JANOTTI-PASSOS, L. K.; SCHALL, V. T. First report on the presence of Biomphalaria straminea in the municipality of Jaboticatubas, state of Minas Gerais, Brazil. Memórias do Instituto Oswaldo Cruz, v. 97, n. 1, p. 27-29, 2002.

7 CAMARGO, E. P. Doenças tropicais. Estudos Avançados, São Paulo, v. 22, n. 64, p. 95-110, dez. 2008.

8 TELES, H. M. S. T. Distribuição geográfica das espécies dos caramujos transmissores de Schistosoma mansoni no Estado de São Paulo. Revista da Sociedade Brasileira de medicina Tropical, Uberaba, v. 38, n. 5, p. 426-432, 2015.

9 CARDIM, L. L.; FERRAUDO, A. S.; PACHECO, S. T. A.; REIS R. B.; SILVA, M. M. N.; CARNEIRO, D. D. M. T.; BAVIA, M. E. Identification of schistosomiasis risk areas using spatial analysis in Lauro de Freitas, Bahia State, Brazil. Cadernos de Saúde Pública, Rio de Janeiro, v. 27, n. 5, p. 899-908, 2011.

10 OHLWEILER, F. P.; EDUARDO, J. M.; TAKAHASHI, F. Y.; CREIN, G. A.; LUCA. L. R.; OLIVEIRA, R. C. Larvas de trematódeos associadas a moluscos de água doce em municípios da Região Metropolitana de São Paulo, Estado de São Paulo, Brasil. Revista Pan-Amaz Saúde, v. 4, n. 3, p. 37-48. 2013.
11 SOUZA, M. A. A.; BARBOSA, V. S.;

ALBUQUERQUE, J. O.; BOCANEGRA, S.; SOUSASANTOS, R.; PAREDES, H.; BARBOSA, C. S. Aspectos ecológicos e levantamento malacológico para identificação de áreas de risco para transmissão da esquistossomose mansoni no litoral norte Pernambuco, Brasil. Iheringia Série Zoologia, v. 100, n. 1, p. 19-24, 2010.

12 LIMA, G. M.; MONTRESOR, L. C.; PONTES, J.; AUGUSTO, R. C.; SILVA, J. P; THIENGO, S. C. Compatibility Polymorphism Based on Long-Term HostParasite Relationships: Cross Talking Between Biomphalaria glabrata and the Trematode Schistosoma mansoni From Endemic Areas in Brazil. Frontiers in Immunology, v. 10, n. 328, p. 1-12, 2019.

13 IBGE - Instituto Brasileiro de Geografia e Estatística, 2016. Disponível em:

<https://cidades.ibge.gov.br/brasil/mg/arcos/panorama $\geq$. Acesso em 08 mai 2018.

14 IBGE - Instituto Brasileiro de Geografia e Estatística, 2017. Disponível em:

<https://cidades.ibge.gov.br/brasil/mg/arcos/panorama 2 . Acesso em 08 mai 2018.

15 PARAENSE, W. L. Estado atual da sistemática dos planorbídeos brasileiros. Arquivos do Museu Nacional do Rio de Janeiro, v. 55, p. 105-128, 1975.

16 BARBOSA, F.S., PINTO, H.A.; MELO, A.L. Biomphalaria straminea (Mollusca: Planorbidae) como hospedeiro intermediário de Zygocotyle lunata (Trematoda: Zygocotylidae) no Brasil. Neotropical Helminthology, v. 5, n. 2, pp. 241-246, 2011.

17 YAMAGUTI, S. Systema helminthum Digenetic Trematodes. New York: Interscience, v. 1, p. 1- 979, 1958.

18 TRAVASSOS, L.; FREITAS, J. F. T.; KOHN, A. Trematódeos do Brasil. Memórias do Instituto Oswaldo Cruz, v. 67, p.1-886, 1969.

19 LÓPEZ-HERNÁNDEZ, D.; LOCKE, S. A.; ASSIS, J. C. A.; DRAGO, F. B.; MELO, A. L.; RABELO, E. M. L.; PINTO, H. A. Morphological and experimentalinfection studies of cercariae of five species in the superfamily Diplostomoidea (Trematoda: Digenea) infecting Biomphalaria straminea (Mollusca: Planorbidae) in Brazil. Acta Tropica, v. 199, p. 1-11, 2019.

20 TIBIRIÇÁ, S. C., GUIMARÃES, F. B.; TEIXEIRA, M. T. B. Schistosoma mansoni in the context of the Brazilian health policy. Ciência \& Saúde Coletiva, v.16, n. 1, p. 375-381, 2011. 
21 COLLEY, D. G.; BUSTINDUY, A. L.; SECOR, W. E.; KING, C. H. Human Schistosomiasis. Lancet, v. 383, n. 9936, p. 2253-2264, 2014.

22 MELO, A. L. \& PEREIRA, L. H. On the finding of Biomphalaria tenagophila naturally infected with Schistosoma mansoni in the state of Minas Gerais, Brasil. Revista do Instituto de Medicina Tropical de São Paulo, v. 27, n. 2, p. 99-101, 1985.

23 TELLES, H.M.S.; PEREIRA, P.A.C.; RICHINITTI, L.M.Z. Distribuição de Biomphalaria (Gastropoda, Planorbidae) nos estados do Rio Grande do Sul e Santa Catarina, Brasil. Revista de Saúde Pública, v. 25, n. 5, p. 350-352, 1991.

24 SOUZA, C.P. et al. Geographical distribution of Biomphalaria snails in the state of Minas Gerais, Brazil. Memórias do Instituto. Oswaldo Cruz, Rio de Janeiro, v. 96, n. 3, p.293-302, 2001.

25 SOUZA, M. A. A.; MELO, A. L. Caracterização de larvas de trematódeos emergentes de moluscos gastrópodes coletados em Mariana, Minas Gerais, Brasil. Iheringia, Série Zoologia, v. 102, n. 1, p. 11-18, 2012.

26 PINTO, A. H.; MELO, A. L. Larvas de Trematódeos em moluscos do Brasil: Panorama e Perspectivas após um século de estudos. Revista de Patologia Tropical, v. 42, n. 4, p. 369-386, 2013.

27 JOURDANE, J., MOUNKASSA, J. B.; IMBERT-

ESTABLET, D. Influence of intramolluscan larval stages of Echinostoma liei on the infectivity of Schistosoma mansoni cercariae. Journal of Helminthology, v. 64, n. 1, p 71-74, 2013.

28 PINTO, A. H.; MELO, A. L. Metacercarie of Centrocestus formosanus (Trematoda: Heterophyidae) in Australoheros facetus (Pisces:Cichlidae) in Brazil.

Revista Brasileira de Parasitologia Veterinária, v. 21, n. 3, p. 334-337, 2012.

29 PINTO, H. A.; GONÇALVES, N. Q.; LÓPEZHERNANDEZ, D.; PULIDO-MURILLO, E. A.; MELO, A. L. The life cycle of a zoonotic parasite reassessed: Experimental infection of Melanoides tuberculata (Mollusca: Thiaridae) with Centrocestus formosanus (Trematoda: Heterophyidae). PLOS ONE, v. 13, n. 4, p. 1-13, 2018.

30 PULIDO-MURILLO, E. A.; FURTADO, L. F. V.; MELO, A. L.; RABELO E. M. L.; PINTO, H. A. Pinto Fishborne Zoonotic Trematodes Transmitted by Melanoides tuberculata Snails, Peru. Emerging Infectious Diseases, v. 24, n. 3, p. 606-609, 2018.

31 PINTO, H. A. Pleurolophocercous and parapleurolophocercous types of cercariae: Revisiting concepts. Parasitology International, v. 68, p. 92-94, 2019.

32 BLASCO-COSTA, I.; POULIN, R. Parasite life-cycle studies: a plea to resurrect an old parasitological tradition. J. Helminthol, v, 91, p. 647656, 2017. 